\title{
Altruísmo Autoinformado e Informado por Pares: Evidências do Altruísmo como Traço de Personalidade
}

\author{
Valdiney Veloso Gouveia \\ Universidade Federal da Paraíba \\ Carlos Eduardo Pimentel \\ Universidade Federal da Paraíba \\ Patrícia Nunes da Fonsêca \\ Universidade Federal da Paraíba \\ Larisse Helena Gomes Macêdo Barbosa \\ Faculdades Integradas de Patos \\ Rildésia Silva Veloso Gouveia \\ Centro Universitário de João Pessoa \\ José Farias de Souza Filho \\ Centro Universitário de João Pessoa
}

\begin{abstract}
RESUMO
Este estudo reuniu evidências acerca do altruísmo como um traço de personalidade. Participaram 438 pessoas, divididas igualmente entre respondentes e seus informantes. Estes responderam os seguintes instrumentos: Escala de Altruísmo Autoinformado, Questionário de Gratidão, Escala de Disposição para Perdoar e Escala de Desejabilidade Social, além de perguntas demográficas; os informantes deram respostas aos instrumentos descrevendo quais eram as características dos respondentes. Os resultados indicaram convergência das estruturas fatoriais e consistência interna da medida de altruísmo, constatando-se correlação entre as medidas auto e heteroinformadas, assim como um padrão de associação congruente de suas pontuações com disposição para perdoar. Concluiu-se que existem evidências do altruísmo como um traço de personalidade, que independe de contexto situacional, estando sua medida dissociada de desejabilidade social.
\end{abstract}

Palavras-chave: altruísmo; personalidade; disposição para perdoar; desejabilidade social.

\begin{abstract}
Self and other-informed altruism: evidence of the altruism as a personality trait

This study aimed to gather evidence on altruism as a personality trait. Participants were 438 people, equally divided into respondents and their informants. They completed the following instruments: Selfinformed Scale of Altruism, Gratitude Questionnaire, Willingness to Forgive Scale, and Social Desirability Scale, in addition to demographic questions. The informants responded as if they were the respondents. Results indicated convergence of the factor structure and reliability of the measure of altruism, verifying correlation between the self and other-informed measures of altruism as well as a consistent pattern of association of their scores with those in willingness to forgive. In conclusion, there is evidence of the altruism as a personality trait, independent of situational context, being its measure dissociated from social desirability.
\end{abstract}

Keywords: altruism; personality; willingness to forgive; social desirability.

Falar de altruísmo é assumir a existência de atitudes genuinamente motivadas, que estão desprovidas de qualquer benefício interno (e.g., melhorar seu autoconceito, autoestima) ou externo (e.g., receber algo em troca), seja de forma direta ou indireta (Abal et al, 2010; Batson \& Powel, 2003). Porém, alguns psicólogos discordam de uma definição precisa acerca deste constru- to, sendo ele tipicamente conceituado como um componente do comportamento pró-social (Batson \& Powel, 2003). Esse tipo de comportamento, por sua vez, é considerado um termo geral das ciências sociais, definido como oposto ao comportamento antissocial (Batson, 1998; Wispé, 1972). De maneira ampla, refere-se a todo e qualquer ato praticado com o objetivo de 
beneficiar outra pessoa ou grupo de pessoas (Eisenberg, 2000), podendo ou não envolver benefícios para o agente, e ser um ato direto ou indireto (Goldstein, 1983).

Dessa forma, o altruísmo não pode ser igualado ao comportamento pró-social, já que este tipo de comportamento apresenta caráter generalista, incluindo uma classe ampla de comportamentos que são benéficos para outrem (Penner, Dovidio, Piliavin, \& Schroeder, 2005). Diferentemente disso, o altruísmo é entendido como um ato pró-social anômalo, específico, raro, atípico e até mesmo extraordinário (Gouveia, Athayde, Gouveia, Gomes, \& Souza, 2010). Alguns pesquisadores entendem este tipo de comportamento como originado apenas em situações específicas. Por exemplo, o estudo empreendido por Latané e Darley (1970) ilustra este ponto ao investigar o motivo para o espectador não responder ou não prestar ajuda diante do assassinato de Katharine "Kitty" Genovese, em 1964, cujo homicídio brutal e morte eventual foi testemunhado por 38 de seus vizinhos em Nova York. Apesar dos apelos e gritos dela, ninguém interviu ou mesmo chamou os policiais. Desde então, esse tema tem sido estudado a fim de encontrar explicações biológicas, motivacionais.

Por outro lado, estudiosos têm enfocado em uma definição de altruísmo como um atributo da personalidade (Diniz, 2009; Gouveia et al; 2011; Gouveia, Santos, Athayde, Souza, \& Gusmão, 2014; Rushton, 1984). De acordo com Rushton, Chrisjohn e Fekken (1981), o altruísmo é uma disposição interna ou um traço de personalidade. Neste sentido, as pessoas não agiriam segundo princípios altruístas apenas em alguns contextos, mas poderiam ser descritas como sendo verdadeiramente altruístas, apresentando comportamentos que revelem esta natureza benevolente e pró-social em diversos contextos.

\section{Altruísmo como Atributo de Personalidade}

Algumas pesquisas sobre os antecedentes do comportamento altruísta o têm definido operacionalmente, isto é, identificando uma conduta específica (e.g., conceder assento em uma sala de espera, ajudar uma pessoa a atravessar a rua), embora diversos pesquisadores costumam conceber o altruísmo como um atributo da personalidade (Krueguer, Hicks, \& Mcgue, 2001; Penner \& Finkelstein, 1998; Steele et al., 2008), buscando sua definição em termos de processos atribucionais e disposicionais. Sob este entendimento, sugere-se que a reação das pessoas diante de uma situação é determinada por um traço latente de personalidade altruísta; logo, este construto seria responsável pela estabilidade do comportamento em diferentes situações (Krebs, 1975; Rushton, 2004). Como traço de personalidade, o altruísmo é definido mais amplamente, cobrindo toda sua dimensão, evitando restringi-lo a comportamentos específicos (Maner \& Gailliot, 2007).

Rushton et al. (1981) trouxeram evidências acerca de diferenças individuais duradouras e congruentes no comportamento altruísta, demonstrando que as pessoas respondem (medidas de autorrelato) consistentemente a diversas situações em que este está implícito. Estes autores consideraram uma amostra de respondentes e informantes (pares, pessoas conhecidas dos respondentes) $(n=416)$, que preencheram a Escala de Altruísmo Autoinformado e quatro perguntas globais sobre o quanto a pessoa considerada era cuidadosa, prestativa, tinha em conta os sentimentos dos demais e se dispunha a fazer um sacrificio por alguém. Seus resultados indicaram que houve congruência na pontuação entre os respondentes e seus pares, demonstrando que indivíduos altruístas são percebidos por seus pares como agindo coerentemente desta forma. Resultados semelhantes foram observados por Carlo, Eisenberg, Troyer, Switzer e Speer (1991).

Posteriormente, Eisenberg et al. (1999) realizaram um estudo longitudinal com crianças de 4 e 5 anos de idade, que foram acompanhadas por cerca de 15 anos. $\mathrm{Na}$ ocasião, estes autores observaram que o altruísmo se observa já na infância, mantendo-se consistente ao longo da vida, provavelmente por esta tendência estar associada com o temperamento e a personalidade, que são, por definição, atributos estáveis. Nesta direção, Krueger, Hicks e Mcgue (2001) realizaram um estudo com intuito de atestar a personalidade altruísta. Para tanto, consideraram a relação dos traços de personalidade com o comportamento antissocial e o altruísmo, averiguando se estes provinham de distintas etiologias ou compreendiam opostos de uma única dimensão. Contando com uma amostra de gêmeos adultos monozigóticos e dizigóticos, eles constataram que o altruísmo e o comportamento antissocial estavam associados fortemente a traços distintos de personalidade, que sugerem etiologias diferentes. Por fim, têm sido realizadas algumas pesquisas que apoiam a proposição de que há uma personalidade altruísta, que é capaz de predizer, por exemplo, o voluntariado e a doação de sangue (Gouveia et al., 2014; Penner \& Finkelstein, 1998; Steele et al., 2008).

Pesquisas nesta direção estão inseridas em uma vertente da Psicologia que trabalha com a questão da ge- 
neralidade, isto é, que primam por conhecer traços que se referem a atributos mais gerais e duráveis das pessoas, como, por exemplo, a personalidade altruísta. Esses estudos geralmente utilizaram escalas ou questionários derivados de medidas de traços de personalidade, relacionando as pontuações dos participantes nestas medidas com algum índice de altruísmo (Diniz, 2009). Neste âmbito, parece oportuno entender como esta medição tem sido feita.

\section{Medindo o Altruísmo}

Para que medir o altruísmo? Trata-se de um comportamento pró-social, de natureza motivada e dirigida a outrem, sem buscar benefícios ou atender a interesses pessoais (Goldstein, 1983; Maner \& Gailliot, 2007). Portanto, se o propósito for conhecer seus antecedentes e consequentes, demandar-se-á medi-lo. Alguns instrumentos foram desenvolvidos para medir as diferenças individuais no comportamento altruísta em adultos (Heist \& Yonge, 1962; Mehrabian \& Epstein, 1972; Rushton et al., 1981) e crianças (Weir \& Duveen, 1981). Observa-se, assim, que as medidas de autorrelato são as mais utilizadas, a exemplo das que desenvolveram O’Connor (2005) (Escala de Disposição Altruísta), focando na disposição altruísta, e Figueredo (2007), que construiu uma bateria com 199 itens para mensurar indicadores cognitivos e comportamentais de estratégias de história de vida. Não obstante, são escassas as informações sobre os parâmetros psicométricos destas medidas (Gouveia et al., 2010).

Rushton et al. (1981) propuseram uma medida de autorrelato do altruísmo, denominada como Escala de Altruísmo Autoinformado (EAA), que tem fundamentado a elaboração de instrumentos específicos de altruísmo, como o proposto por Smith (2006). A EAA vem se apresentando como psicometricamente adequada em diversas culturas (Gouveia et al., 2010; Krueger et al., 2001; Mclean, Walker, \& Matsuba, 2004). Trata-se de uma medida tipo lápis e papel, autoaplicável, composta por 20 itens (e.g., Já ofereci ajuda a um deficiente ou idoso desconhecido para atravessar a rua), respondidos em escala Likert de cinco pontos, variando de 0 (Nunca) a 4 (Muito frequentemente). Originalmente elaborada em contexto canadense (Ontário), considerou cinco amostras de estudantes universitários somando um total de 611 participantes $\left(N_{1}=99, N_{2}=56, N_{3}=\right.$ $118, N_{4}=146$ e $N_{5}=192$ ), sendo a maioria do sexo feminino (60,4\%). Admitindo estrutura unifatorial, mostrou consistência interna (alfa de Cronbach) aceitável, variando de $0,78\left(N_{3}\right)$ a $0,87\left(N_{4}\right)$, com alfa médio de
0,84 .

Observaram-se também evidências de validade preditiva da EAA, correlacionando suas pontuações com oito indicadores de altruísmo, cinco dos quais implicavam ter realizado um comportamento: ler para uma pessoa cega em resposta a uma solicitação telefônica; participar voluntariamente em um experimento de um pesquisador que necessitava de colaborador; ter feito curso de primeiros socorros; ter preenchido uma carteira de doador de órgão, que é anexada à licença de motorista em Ontário; e ter interesse de ajudar; os outros três indicadores abarcavam pontuações em escalas medindo construtos teoricamente similares: atitudes sensitivas; traço de cuidado / atenção; e presteza em cenário de urgência. As pontuações da EAA se correlacionaram diretamente com o conjunto destes indicadores $(r=$ $0,40, p<0,01)$, sendo mais forte $(p<0,01)$ com os seguintes: atitudes sensitivas $(r=0,33)$, traço de cuidado $(r=0,28)$ e doação de órgãos $(r=0,25)$.

Destaca-se que essa escala foi adaptada para o contexto brasileiro por Gouveia et al. (2010), os quais procuraram reunir evidências de sua validade fatorial e consistência interna. Na ocasião, observaram uma estrutura unifatorial, cujos indicadores de ajuste foram considerados satisfatórios (e.g., $G F I=0,92, R M S E A=$ $0,058)$; esta medida obteve alfa de Cronbach acima do comumente recomendado (Pasquali, 2003), situando-se em 0,83 , tendo a homogeneidade média dos itens (correlação item-total) ficado em 0,28. Portanto, esta parece ser um instrumento promissor para avaliar o altruísmo, justificando seu emprego em pesquisas neste contexto.

Por fim, este estudo objetiva oferecer evidências acerca do altruísmo como um traço de personalidade, não se restringindo a uma resposta situacional. Neste sentido, a convergência das pontuações dos participantes e seus pares (informantes, pessoas que, conhecendo os participantes, respondem o instrumento como se fossem eles) reforça a concepção de que o altruísmo pode ser percebido como uma disposição ou um traço de personalidade que serve para descrever a forma como as pessoas costumam se comportar em sua vida quotidiana. Portanto, espera-se que haja convergência nas estruturas fatoriais da medida de altruísmo nos dois grupos (respondentes e informantes), tal como ocorreu no estudo de Rushton et al. (1981). Confia-se, ainda, que as pontuações de altruísmo nesses dois grupos apresentem padrão similar de associação com três construtos distintos: disposição para perdoar, gratidão e desejabilidade social, que são aferidos por instrumentos específicos. 
A disposição para perdoar é a capacidade de agir consistentemente de modo completo e irrestritamente disposto a oferecer o perdão, independentemente do contexto. McCullough, Worthington e Rachal (1997) consideram o perdão e o altruísmo como motivados pelo sentimento empático, expressando a superação de interesses pessoais, que é típico de alguém que age de forma desprendida, sem necessariamente esperar algo em troca (Worthington Jr., Sharp, \& Lerner, 2006). No que se refere à gratidão, compreende um sentimento reativo ou retributivo de um indivíduo em resposta a uma ação generosa (Westermarck, 1928); refere-se, deste modo, ao reconhecimento de ações dos demais que favoreceram o indivíduo, sendo uma espécie de reação instrumental ao altruísmo (McCullough, Kimeldorf, \& Cohen, 2008), não necessariamente implicando em um ato altruísta em si (McCullough, Emmons, \& Tsang, 2002). Por fim, a desejabilidade social é um viés de resposta e um traço de personalidade, expressando tendência a se mostrar de forma positiva, politicamente correta; indivíduos com pontuações altas em desejabilidade social podem procurar pontuar alto em altruísmo, favorecendo questionar a validade das medidas deste construto (Chou, 1996). Deste modo, em vários estudos sobre o altruísmo a desejabilidade é avaliada, checando a necessidade de controlá-la (Carlo et al., 1991; Kruger, 2003; Rosenhan, Solovey, \& Hargis, 1981; Nickell, 1998).

Em razão do anteriormente descrito, estima-se que a maior correlação do altruísmo será com o primeiro construto, considerando inerente ao ato de perdoar a dimensão de superação dos interesses pessoais que caracteriza o referido traço de personalidade (Worthington Jr. et al., 2006). No caso da gratidão, sua correlação com o altruísmo poderá ser negativa ou mesmo nula; aquele que age de forma altruísta pode não esperar o reconhecimento, a gratidão. Por fim, seria recomendável que a medida de altruísmo não se correlacionasse com aquela de desejabilidade social, descartando a possibilidade de um viés de resposta na EAA, indicando evidências de sua validade discriminante.

Diante do que foi exposto, no estudo em questão a ênfase recai no altruísmo por reconhecer seu potencial para a explicação de alguns comportamentos sociais importantes (Blanca, Rando, Frutos, \& López-Montiel, 2007; Loureiro, 2010; Rushton et al., 1981) e, sobretudo, pela escassez de pesquisas a respeito no Brasil. Especificamente, objetiva-se comparar a estrutura fatorial da Escala de Altruísmo Autoinformado (Rushton et al.,1981), considerando quando o altruísmo é autoinfor- mado (o próprio respondente) ou informado por pares (amigos ou familiares do respondente). Se é certo que o altruísmo compreende uma dimensão de personalidade, então a estrutura que emergirá não variará se o informante for a própria pessoa ou alguém que a conheça, e as pontuações correspondentes apresentarão aproximadamente os mesmos padrões de correlação com outras variáveis. Estas foram as hipóteses principais que orientaram este estudo.

\section{MÉTODO}

\section{Participantes}

Participaram 438 pessoas igualmente distribuídas entre respondentes $\left(N_{1}\right)$ e seus informantes $\left(N_{2}\right)$. Os integrantes da primeira amostra tinham idade média de 22,6 anos ( $D P=7,24$, variando de 16 a 55 anos), sendo a maioria do sexo feminino $(79,9 \%)$; e seus informantes tinham idade média de 26,1 anos $(D P=11,23$, variando de 12 a 72 anos), sendo majoritariamente do sexo feminino (67\%). Estas compreenderam amostras de conveniência (não-probabilísticas), participando as pessoas que, contatadas, decidiram colaborar.

\section{Instrumentos}

Os participantes foram solicitados a responder os seguintes instrumentos:

Escala de Altruísmo Autoinformado (EAA). Desenvolvida por Rushton et al. (1981), consta de 20 itens que expressam comportamentos que a pessoa possa ter realizado (e.g., Já doei bens ou roupas para uma organização de caridade; Já segurei um elevador e mantive a porta aberta para que um estranho pudesse entrar). O participante é demandado a indicar se já apresentou cada um dos comportamentos indicados, respondendo em escala de cinco pontos, variando de 0 (Nunca) a 4 (Muito frequentemente). Os parâmetros psicométricos da versão em português foram previamente detalhados.

Questionário de Gratidão (QG). Esta é uma medida curta, composta por seis itens (e.g., Eu tenho muita coisa na vida que agradecer; Sou grato a um número grande de pessoas), construída inicialmente para o contexto estadunidense (McCullough et al., 2002). O participante é solicitado a informar em que medida está de acordo ou em desacordo com cada afirmação, empregando uma escala de resposta de sete pontos, variando de 1 (Discordo totalmente) a 7 (Concordo totalmente). Seus autores reuniram evidências de que esta é uma medida unifatorial, com alfa de Cronbach médio (considerando cinco amostras) superior a 0,80, 
apresentando validade convergente com satisfação com a vida, afetos positivos e negativos, ansiedade, depressão, preocupação empática e transcendência espiritual. Sua adaptação ao contexto brasileiro foi realizada por Aquino (2009), quem corroborou a natureza unidimensional desta medida.

Escala de Disposição para Perdoar (EDP). Elaborada por DeShea (2003) no contexto estadunidense, é composta por 12 cenários frente aos quais o respondente precisa indicar o quanto estaria disposto a perdoar a pessoa (o ator) que é destacado (e.g., Você comenta com um colega de trabalho, Cristiano, uma ideia que você teve que melhoraria a eficiencia e reduziria os gastos da empresa. Durante uma reunião com os trabalhadores da empresa, o diretor anuncia a implementação da referida ideia e diz que a pessoa que a sugeriu, Cristiano, receberá um bônus de $R \$ 1.000$ pela ideia). As respostas são dadas em escala de sete pontos, variando de 0 (Nada disposto) a 6 (Completamente disposto). Esta autora observou uma estrutura unifatorial, apresentando alfas de Cronbach próximos a 0,90, tendo sido observados resultados similares no Brasil (Gouveia et al., 2009).

Escala de Desejabilidade Social (EDS). Proposta por Crowne e Marlowe (1960), esta escala expressa atos que podem indicar uma necessidade de aprovação por parte dos participantes, sendo formado por 33 itens (e.g., Nunca hesito em sair do meu caminho para ajudar alguém em dificuldades; Algumas vezes já desisti de fazer algo porque não confiava na minha capacidade). Para respondê-los, o participante deve marcar $V$ (Verdadeiro) ou $F$ (Falso), de acordo com seu comportamento; quanto maior o valor, maior a tendência à desejabilidade social. Seus autores propuseram uma estrutura unidimensional, cujo coeficiente $K R-20$ foi de 0,88 ; sua versão brasileira também apresentou uma solução unifatorial (alfa de Cronbach de 0,79) (Ribas Jr, Moura, \& Hutz, 2004). Nesse estudo emprega-se a versão reduzida, com 20 itens, descrita por Gouveia, Guerra, Sousa, Santos e Costa (2009), que corroboraram a estrutura unifatorial proposta, que apresentou consistência interna satisfatória $(K R-20=0,76)$.

Caracterização dos Participantes. Incluíram-se cinco perguntas demográficas (sexo, idade, estado civil, religião e o quanto ele se considera religioso.

\section{Procedimento}

No caso dos participantes, os questionários foram aplicados em ambiente coletivo de sala de aula, porém respondidos individualmente. Três colaboradores fica- ram responsáveis pela coleta, que foram levadas a cabo conforme disponibilidade dos professores que cederam suas salas de aula. Após se apresentarem aos participantes, informando sobre o estudo, solicitou-se que colaborassem voluntariamente; ademais, houve o pedido para que também pudessem solicitar que uma pessoa próxima (familiar ou amigo íntimo) respondesse ao questionário, porém tomando em conta a descrição do próprio comportamento do participante, isto é, a instrução indicava que os informantes deveriam responder as escalas pensando na pessoa que solicitou sua participação. No caso daqueles que concordaram em obter a resposta de seu informante, entregou-se o questionário correspondente, que deveria ser respondido e devolvido no prazo de três dias. A ordem de apresentação dos instrumentos em cada questionário foi contrabalanceada, evitando efeito de exposição, isto é, que as respostas à primeira escala influenciassem consistentemente àquelas das subsequentes. Os participantes foram orientados a não assinarem ou se identificarem no questionário, assegurando o anonimato de sua participação. Para isso, os instrumentos foram codificados em pares. A todos foi assegurado o caráter voluntário de sua contribuição. Esta pesquisa recebeu parecer favorável do Comitê de Ética do Hospital Universitário Lauro Wanderley (Protocolo CEP/HULW no 147/10).

\section{Análise dos Dados}

Para a tabulação e as análises dos dados foi utilizado o $P A S W$ (versão 18). Inicialmente, realizaram-se análises de componentes principais para as amostras de participantes e informantes, procurando extrair uma estrutura unifatorial comum. Em seguida, procedeu-se o cômputo da congruência fatorial. Esta técnica permite avaliar a estabilidade dos resultados do modelo fatorial (Hair, Anderson, Tatham, \& Black, 2009), referindo-se ao grau de congruência (cosseno do vetor) entre dois conjuntos de cargas fatoriais (Gorsuch, 1983). Portanto, é realizada quando se faz necessário comparar as estruturas fatoriais obtidas em dois estudos, considerando o mesmo conjunto de itens. Para cada estrutura foi calculado seu respectivo coeficiente de consistência interna (alfa de Cronbach), os quais foram comparados por meio do teste de Hakstian-Whalen (MH-W; Kim \& Feldt, 2008). Por fim, foram realizadas análises de correlação ( $r$ de Pearson) tanto entre as pontuações em altruísmo dos respondentes e seus informantes, como com os construtos disposição para perdoar, gratidão e desejabilidade social, visando oferecer evidências adicionais de que o altruísmo não se restringe a respostas 
situacionais, mas apresenta-se como um traço de personalidade.

\section{RESULTADOS}

Primeiramente, calcularam-se análises de componentes principais para as amostras de respondentes $\left[\chi^{2}\right.$ $(190)=1.161,55, p<0,001 ; K M O=0,88]$ e seus informantes $\left[\chi^{2}(190)=1.029,75, p<0,001 ; K M O=0,85\right]$. $\mathrm{Na}$ amostra de respondentes, empregando o critério de Kaiser (valor próprio igual ou superior a 1), até cinco componentes poderiam ser extraídos (valores próprios: $6,54,1,57,1,28,1,17$, e 1,02), explicando conjuntamente $58,0 \%$ da variância total; o fator geral explicou $56,4 \%$ desta variância. Na amostra de informantes, empregando-se o mesmo critério, seria possível identificar cinco componentes (valores próprios: 5,91, 1,67, 1,38, $1,18$ e 1,00$)$, os quais explicaram $55,7 \%$ da variância total, cabendo ao fator geral contribuir com $53,1 \%$ desta variância. Foi realizada ainda análise paralela (critério de Horn), que sugeriu dois e três fatores, respectivamente. Contudo, consideraram-se os achados prévios acerca da unidimensionalidade desta medida, que mostrou melhor interpretável (Gouveia et al., 2010; Rushton et al., 1981). Deste modo, decidiu-se extrair um único componente, sendo os resultados apresentados na Tabela 1.

As saturações para a amostra de respondentes variaram de 0,31 [Item 5. Já dei dinheiro para um estranho necessitado (ou que me pediu)?] a 0,68 (Item 2. Já dei direções ou orientações a um estranho?), com média de 0,56; no caso da amostra de informantes, estas variaram de 0,25 (Item 8. Já doei sangue?) a 0,65 (Item 18. Já ofereci ajuda a um deficiente ou idoso desconhecido para atravessar a rua?), com média de 0,54.

Com o fim de checar se poderia ser extraída uma mesma estrutura fatorial, procedeu-se ao cômputo da congruência fatorial das soluções apresentadas previamente, observando-se valor de 0,98 . Portanto, confirma-se a pertinência de contar com uma estrutura fatorial comum, apresentando uma única dimensão. Nesta mesma direção, procurou-se calcular para cada amostra o alfa de Cronbach do fator geral correspondente, reunindo todos os 20 itens, obtendo-se os seguires coeficientes: 0,88 e 0,87 , respectivamente. $O$ teste de Hakstian-Whalen permitiu comprovar que tais valores não foram estatisticamente diferentes $(\mathrm{MH}-\mathrm{W}=$ $3,05, p>0,05)$, reforçando a concepção de um traço de personalidade inerente à medida de altruísmo.

Preliminarmente, antes de apresentar as correlações de altruísmo com os outros construtos, pareceu pertinente descrever o desempenho das amostras nas medidas efetuadas. Nessa direção, computaram-se as pontuações médias dos respondentes e seus informantes em altruismo, disposição para perdoar e gratidão. No que diz respeito ao altruísmo, os respondentes obtiveram pontuação média de $1,53(D P=0,68)$ e os informantes de $1,53(D P=0,61)$ [variação da medida de 0 (Nunca) a 4 (Muito frequentemente)]; em relação à disposição para perdoar, a pontuação média dos participantes foi de 2,61 $(D P=1,14)$ e dos informantes de $2,61(D P=1,18)$ [variação da medida de 0 (Nada disposto) a 6 (Completamente disposto)]; e, por fim, na medida de gratidão a pontuação média dos participantes foi de 4,89 $(D P=0,71)$ e dos informantes de 4,64 (DP $=0,74$ ) [amplitude da medida de 1 (Discordo totalmente) a 7 (Concordo totalmente)]. Portanto, os participantes e seus informantes apresentaram pontuações baixas em altruísmo e disposição para perdoar, sendo mais elevadas aquelas em gratidão.

Por fim, procurou-se conhecer a congruência nas pontuações totais da medida de altruísmo auto (respondentes) e heteroinformado (informantes), assim como em que medida estas se correlacionam com outros construtos (disposição para perdoar, gratidão e desejabilidade social), favorecendo pensar em evidências de validade convergente-discriminante. Os resultados a respeito são apresentados na Tabela 2 .

Como é possível observar, a correlação das pontuações dos respondentes e seus informantes em altruísmo foi significativa e positiva, inclusive correspondendo ao maior coeficiente de correlação $(r=0,40, p<0,001)$. No caso das pontuações dos informantes, o altruísmo se correlacionou diretamente com a disposição para perdoar $(r=0,18, p<0,05) \mathrm{e}$, inversamente, a gratidão $(r$ $=-0,30, p<0,01)$. Quanto às pontuações dos respondentes em altruísmo, unicamente se correlacionaram com a disposição para perdoar $(r=0,28, p<0,001)$. Ressalta-se, por último, que a medida de altruísmo não se correlacionou com a desejabilidade social nas amostras de respondentes e seus informantes $(r<0,10)$.

\section{DISCUSSÃO}

Presumindo que o altruísmo compreende um traço de personalidade (Rushton et al., 1981), este estudo objetivou reunir evidências a respeito. Precisamente, pretendeu comprovar a convergência das pontuações de respondentes e seus informantes neste traço. No caso, checaram-se a congruência das estruturas fatoriais, a 
Tabela 1

Estrutura fatorial da EAA nos Grupos de Participantes e Informantes

\begin{tabular}{|c|c|c|c|}
\hline \multirow{2}{*}{ Item } & \multirow{2}{*}{ Descrição } & \multicolumn{2}{|c|}{ Fator Geral } \\
\hline & & Respondentes & Informantes \\
\hline 02 & Já dei direções ou orientações a um estranho. & 0,68 & 0,59 \\
\hline 16 & $\begin{array}{l}\text { Já ajudei um(a) colega de classe, que não conheço muito bem, com um trabalho da } \\
\text { faculdade quando meu conhecimento era maior que o dele(a). }\end{array}$ & 0,67 & 0,45 \\
\hline 14 & $\begin{array}{l}\text { Já deixei um vizinho, que não conheço muito bem, pegar emprestado algo de valor (por } \\
\text { exemplo, ferramentas, eletrodomésticos). }\end{array}$ & 0,65 & 0,59 \\
\hline 19 & Já ofereci meu assento no ônibus para um desconhecido que estava de pé. & 0,65 & 0,56 \\
\hline 01 & Já ajudei a empurrar um carro enguiçado (quebrado) de um estranho & 0,64 & 0,51 \\
\hline 18 & Já ofereci ajuda a um deficiente ou idoso desconhecido para atravessar a rua. & 0,63 & 0,65 \\
\hline 13 & $\begin{array}{l}\text { Já mostrei a um balconista (por exemplo, no supermercado, na lanchonete) seu erro por ter } \\
\text { me cobrado menos do que eu deveria pagar. }\end{array}$ & 0,62 & 0,54 \\
\hline 11 & $\begin{array}{l}\text { Já deixei alguém passar na minha frente em uma fila (na fotocopiadora ou no } \\
\text { supermercado). }\end{array}$ & 0,61 & 0,61 \\
\hline 09 & Já ajudei a carregar os pertences de um estranho (livros, sacolas, etc.). & 0,61 & 0,59 \\
\hline 07 & Já trabalhei como voluntário para uma organização de caridade. & 0,60 & 0,64 \\
\hline 12 & Já dei carona a um estranho no meu carro (dos meus pais ou amigos). & 0,60 & 0,44 \\
\hline 04 & Já dei dinheiro para uma organização de caridade. & 0,55 & 0,55 \\
\hline 15 & $\begin{array}{l}\text { Já comprei cartões de Natal de organizações de caridade só por saber que se tratava de } \\
\text { uma boa causa. }\end{array}$ & 0,55 & 0,51 \\
\hline 20 & Já ajudei um conhecido a mudar de casa. & 0,53 & 0,60 \\
\hline 03 & Já troquei dinheiro para um estranho. & 0,53 & 0,52 \\
\hline 10 & Já segurei um elevador e mantive a porta aberta para que um estranho pudesse entrar. & 0,51 & 0,50 \\
\hline 17 & $\begin{array}{l}\text { Já fui solicitado, voluntariamente, para tomar conta de animais de estimação ou crianças do } \\
\text { vizinho sem receber qualquer pagamento em troca. }\end{array}$ & 0,48 & 0,50 \\
\hline 06 & Já doei bens ou roupas para uma organização de caridade. & 0,42 & 0,53 \\
\hline 08 & Já doei sangue. & 0,42 & 0,25 \\
\hline 05 & Já dei dinheiro para um estranho necessitado (ou que me pediu). & 0,31 & 0,62 \\
\hline Valor $\mathrm{P}$ & óprio & 6,54 & 5,91 \\
\hline$\%$ Vari & cia Total & 32,7 & 29,6 \\
\hline alfa de & ronbach & 0,88 & 0,87 \\
\hline
\end{tabular}

correlação das pontuações no fator resultante e seu padrão de correlação com três variáveis (disposição para perdoar, gratidão e desejabilidade social). Estima-se, portanto, que esse objetivo tenha sido alcançado.
Apesar do anteriormente indicado, reconhecem-se limitações potenciais deste estudo. Por exemplo, consideraram-se amostras de conveniência, bastante específicas, tendo em conta estudantes universitários e

Tabela 2.

Correlatos do Altruísmo Auto e Heteroinformado

\begin{tabular}{lllll}
\multicolumn{1}{c}{ Variáveis } & \multicolumn{1}{c}{$\mathbf{1}$} & \multicolumn{1}{c}{$\mathbf{2}$} & \multicolumn{1}{c}{$\mathbf{3}$} & \multicolumn{1}{c}{$\mathbf{4}$} \\
\hline 1. Altruísmo & $\mathbf{0 , 4 0 ^ { * * * }}$ & $0,18^{*}$ & $-0,30^{* * *}$ & 0,07 \\
2. Disposição para perdoar & $0,28^{* * *}$ & $\mathbf{0 , 2 1 ^ { * * }}$ & 0,07 & $0,31^{* * *}$ \\
3. Gratidão & 0,04 & 0,01 & $\mathbf{0 , 3 0 * *}$ & 0,01 \\
4. Desejabilidade Social & 0,02 & $0,18^{*}$ & $-0,10$ & $\mathbf{0 , 2 1 ^ { * * }}$ \\
\hline
\end{tabular}

Nota: ${ }^{*} p<0,05,{ }^{* *} p<0,01,{ }^{* * *} p<0,001$ (teste bicaudal). A parte inferior da tabela representa os correlatos do grupo de informantes, enquanto a parte superior descreve os correlatos do grupo de respondentes; os valores da diagonal representam as correlações entre os grupos de respondentes e informantes. 
pessoas de seu convívio (amigos e familiares), que podem não representar a população brasileira ou mesmo a paraibana. Contudo, ressalta-se que não foi o intento generalizar os resultados, mas tão somente reunir evidências de que o altruísmo, como medido pela Escala de Altruismo Autoinformado, configura-se como um traço de personalidade. Outra limitação potencial foi o fato de os respondentes levarem o questionário para ser respondido por seus pares em casa, talvez favorecendo que os informantes os avaliassem de modo mais favorável do que poderiam ser. Porém, convém resaltar que nas duas amostras não houve correlações com desejabilidade social. Por fim, cabe destacar que as amostras foram predominantemente femininas; quiçá se a composição fosse diferente os resultados poderiam variar, ao menos o altruísmo costuma ser maior entre mulheres (Andreoni \& Vesterlund, 2001; Eckel \& Grossman, 1998).

Feitas as ponderações prévias, cabe discutir os achados principais deste estudo. Conforme o conjunto de achados descritos previamente, reuniram-se evidências favoráveis à existência de um traço de personalidade denominado altruísta. Algumas pessoas são consistentemente mais generosas, prestativas e gentis do que outras, o que faz com que sejam prontamente percebidas e descritas como altruístas. Cumpre salientar que mesmo se tratando de um traço de personalidade, sua percepção pode variar quando se trata de uma avaliação dos pares, pois as pessoas podem perceber de forma diferente as ações de outras. Deste modo, coerentemente, de acordo com Rushton et al. (1981), o altruísmo se apresenta como uma disposição interna ou um traço de personalidade; as pessoas não agiriam segundo princípios altruístas apenas em alguns contextos, mas poderiam ser descritas como sendo verdadeiramente altruístas, apresentando comportamentos que revelem esta natureza benevolente e pró-social.

Reforça essa concepção os achados relatados, a exemplo da congruência da estrutura fatorial da Escala de Altruísmo Autoinformado (Rushton et al., 1981), que emerge como unifatorial nos grupos de respondentes e seus informantes, sugerindo que não se trata apenas de agir de modo altruísta, revelando uma resposta situacional, mas ser altruísta, refletindo um traço de personalidade. A propósito, o coeficiente de congruência próximo a 1 sugere estruturas "altamente similares" (Andrade \& Flores-Mendoza, 2010; Jensen, 1998). Não apenas os itens individualmente apresentaram saturações que se correlacionaram, mas também a propriedade de consistência interna desta medida (alfa de
Cronbach) foi bastante similar (Kim \& Feldt, 2008), estando, inclusive, acima de coeficientes proclamados na literatura (Lance, Butts, \& Michels, 2006). Na mesma direção, constatou-se a correlação entre as pontuações na medida de altruísmo dos respondentes e seus informantes, que pode ser considerada forte quando observados os coeficientes comumente encontrados em Psicologia (Gouveia, Santos, \& Milfont, 2009).

Quando foram consideradas variáveis externas ao construto altruísmo, o padrão de convergência pareceu evidente ao comparar os resultados para os respondentes e seus informantes. Desta forma, como esperado, as pontuações em altruísmo se correlacionaram mais fortemente com aquelas em disposição para perdoar, que expressa a superação de interesses pessoais, passando por cima de rixas e eventuais danos sofridos, que é típico de alguém que age de forma desprendida, sem necessariamente esperar algo em troca (Worthington Jr., Sharp, \& Lerner, 2006). A gratidão, que indica o reconhecimento por ações dos demais que favoreceram o indivíduo, não é em si uma dimensão do altruísmo (McCullough et al., 2002), pois apenas expressa uma reação a atos desta natureza ou quaisquer outros que tenham beneficiado o indivíduo (McCullough et al., 2008). Por certo, no presente estudo sua pontuação foi negativamente correlacionada com o altruísmo entre os respondentes, quiçá indicando que a pessoa altruísta desestima a conduta de gratidão, provavelmente por estar inerente a "obrigação" do reconhecimento de uma ação que pode ser genuinamente altruísta. Por fim, coerente com o esperado, embora o altruísmo compreenda um padrão de conduta socialmente estimulado, como foi avaliado, não se correlacionou com a desejabilidade social, que sugere uma disposição a se mostrar agradável aos demais (Dodaj, 2012). Isso não apenas indica a especificidade do altruísmo, como reforça evidências de validade discriminante da medida empregada.

Em suma, diante dos resultados, pode-se concluir que existem indícios de que o altruísmo não se limita a uma resposta situacional (Rushton et al., 1981). Contrariamente, entende-se que se trata de um traço de personalidade, segundo a convergência constatada nos grupos de respondentes e informantes em diferentes aspectos (estruturas da medida, coeficientes de consistência interna, pontuações em altruísmo e sua correlação com outras variáveis). Portanto, trata-se de uma disposição, um traço de personalidade que serve para descrever a forma como as pessoas costumam se comportar na sua vida quotidiana. 


\section{CONSIDERAÇÕES FINAIS}

Pode-se concluir que existem evidências para se considerar o altruísmo como um traço de personalidade, constituindo um construto responsável por um comportamento consistente em diferentes situações. Contudo, é importante que esta pesquisa seja replicada em outros contextos; apesar de ser considerado um país coletivista (Gouveia, Albuquerque, Clemente, \& Espinosa, 2002), o Brasil possui diferentes regiões, cada qual apresentando suas especificidades. Neste sentido, as pessoas poderiam se diferenciar em função do contexto cultural que compartilham. A propósito, é mais comum que em cidades interioranas as pessoas estejam mais voltadas para o outro e, consequentemente, podem estar mais dispostas a ajudar do que aquelas que vivem em grandes capitais, cujo ritmo de vida acelerado leva a uma redução do contato social (Hofstede, Hofstede, \& Minkov, 2010). Poder-se-ia, ainda, empreender esforços para contar com amostras maiores e mais heterogêneas, incluindo pessoas de diferentes níveis econômicos e educacionais.

Por fim, não se trata, apenas, de replicar este estudo, mas procurar conhecer algo mais a respeito do traço altruísmo. Neste sentido, embora previamente tenha sido mencionado o aspecto da influência cultural, cabe ponderar igualmente a influência de fatores genéticos na sua constituição; os estudos a respeito têm sido controversos. Por exemplo, Rushton (2004) aponta que a genética apresenta uma contribuição maior do que o ambiente familiar compartilhado. Por outro lado, Krueger et al. (2001) indicam maior influência de um processo de transmissão cultural, envolvendo o compartilhamento do ambiente familiar e não familiar, do que de base genética para a constituição do altruísmo. Será importante, também, pensar em estudos futuros com o fim de conhecer o processo de desenvolvimento da personalidade

\section{REFERÊNCIAS}

Abal, F. J. P., Lozzia, G. S., Blum, D., Galibert, M. S., Aguerri, M. E., \& Attorresi, H. F. (2010). Análisis de ítems de un test de altruismo a partir del modelo logístico de um parámetro. Perspectivas en Psicología, l7, 16-23.

Andrade, A. C., \& Flores-Mendoza, C. (2010). Transtorno do déficit de atenção/hiperatividade: $\mathrm{O}$ que nos informa a investigação dimensional? Estudos de Psicologia, 15, 1724.

Andreoni, L. \& Vesterlund, L. (2001). Which is the fair sex? Gender differences in altruism. Quarterly Journal of Economics, 116, 293-312.
Aquino, T. A. A. (2009). Atitudes e intenções de cometer o suicídio: Seus correlatos existenciais e normativos. Tese de Doutorado. Departamento de Psicologia, Universidade Federal da Paraíba, João Pessoa, PB.

Batson, C. D. (1998). Altruism and prosocial behavior. In D.T. Gilbert \& S.T. Fiske (Eds.), Handbook of social psychology (pp. 282-316). Boston, MA: McGraw-Hill.

Batson, C. D., \& Powell, A. A. (2003). Altruism and prosocial behavior. In T. Millon, M. J. Lerner \& I. B. Weiner (Eds.), Handbook of psychology: Personality and social psychology (Vol. 5, pp. 463-484). New York: John Wiley \& Sons, Inc.

Blanca, M. J., Rando, B., Frutos, M. A., \& López-Montiel, G. (2007). Perfil psicológico de potenciales donantes y no donantes de órganos. Psicothema, 19, 440-445.

Call, J. (2012). Prosociabilidad y altruismo en nuestros parientes más próximos. Revista de la Sociedad Española de Biología Evolutiva, 7, 25-31.

Carlo, G., Eisenberg, N., Troyer, D., Switzer, G., \& Speer, A. L. (1991). The altruistic personality: In what contexts is it apparent? Journal of Personality and Social Psychology, $61,450-458$.

Crowne, D. P., \& Marlowe, D. (1960). A new scale of social desirability independent of psychopathology. Journal of Consulting Psychology, 24, 349-354.

DeShea, L. A. (2003). Scenario-based Scale of Willingness to Forgive. Individual Differences Research, 1, 201-217.

Diniz, P. K. C. (2009). Correlatos valorativos e emocionais do altruísmo. Dissertação de Mestrado, Universidade Federal da Paraíba, Departamento de Psicologia, João Pessoa, PB.

Dodaj, A. (2012). Social desirability and self-reports: Testing a content and response-style model of socially desirable responding. Europe's Journal of Psychology, 8, 651-666.

Eckel, C. C., \& Grossman, P. J. (1998). Are women less selfish than men?: Evidence from dictator experiments. The Economic Journal, 108, 726-735.

Eisenberg, N. (2000). Emotion, regulation, and moral development. Annual Review Psychology, 51, 665-697.

Eisenberg, N., Guthrie, I. K., Muphy, B. C., Shepard, S. A., Cumberland, A., \& Carlo, G. (1999). Consistency and development of prosocial dispositions: A longitudinal study. Child Development, 70, 1360-1372.

Figueredo, A. J. (2007). The Arizona life history battery. Endereço da página Web: http://www.u.arizona.edu/ ajf/alhb.html (consultado em 19 de setembro de 2011).

Goldstein, J. (1983). Psicologia social. Rio de Janeiro: Guanabara.

Gorsuch, R. (1983). Factor analysis. Mahwah, NJ: Lawrence Erlebaum Associates.

Gouveia, V. V., Athayde, R. A. A. A., Gouveia, R. S. V., Gomes, A. I. A. S. B., \& Souza, R. V. L. (2010). Escala de Altruísmo Autoinformado: Evidências de validade de construto. Aletheia, 33, 30-45.

Gouveia, V.V., Albuquerque, F. J. B., Clemente, M., \& Espinosa, P. (2002). Human values and social identities: A study in two collectivist cultures. International Journal of 
Psychology, 37, 333-342.

Gouveia, V.V., Costa, J. M., Araújo, L.B.U., Gouveia, R.S.V., Medeiros, E.D., \& Gonçalves, M.P. (2009). Disposição para perdoar, desejabilidade social e religião: Um estudo correlacional. Revista Bioética, 17, 297-308.

Gouveia, V. V., Guerra, V. M., Sousa, D. M. F. de, Santos, W. S., \& Costa, J. de M. (2009). Escala de desejabilidade social de Marlowe-Crowne: Evidências de sua validade fatorial e consistência interna. Avaliação Psicológica, 8, 87-98.

Gouveia, V. V., Santos, W. S., Athayde, R. A. A., Souza, R. V. L., \& Gusmão, E. E. S. (2014). Valores, altruísmo e comportamentos de ajuda: Comparando doadores e não doadores de sangue. Psico-PUCRS, 45, 209-218.

Gouveia, V. V., Santos, W. S., \& Milfont, T. L. (2009). O uso da estatística na avaliação psicológica: comentários e considerações práticas. In C. S. Hutz (Org.), Avanços e polêmicas em avaliação psicológica: em homenagem a Jurema Alcides Cunha (pp. 127-155). São Paulo: Casa do Psicólogo.

Hair, J. F. Jr., Anderson, R. E., Tatham, R. L., \& Black, W. C. (2009). Análise multivariada. Porto Alegre, RS: Bookman.

Hofstede, G., Hofstede, G. J., \& Minkov, M. (2010). Cultures and organizations: Software of the mind ( $3^{\mathrm{a}} \mathrm{ed}$.). New York: McGraw-Hill.

Jensen, A. (1998). The g factor: The science of mental ability. London: Praeger.

Kim, S., \& Feldt, L. S. (2008). A comparison of tests for equality of two or more independent alpha coefficients. Journal of Educational Measurement, 45, 179-193.

Krebs, D. L. (1975). Empathy and altruism. Journal of Personality and Social Psychology, 32, 1134-1146.

Krueger, R. F., Hicks, B. M., \& Mcgue, M. (2001). Altruism and antisocial behavior: Independent tendencies, unique personality correlates, distinct etiologies. Psychology Science, 12, 397-402.

Lance, C. E., Butts, M. M., \& Michels, L. C. (2006). The sources of four commonly reported cutoff criteria: What did they really say? Organizational Research Methods, 9, 202-220.

Latané, B., \& Darley, J. M. (1970). The unresponsive bystander: Why doesn't he help? Englewood Cliffs, NJ: Prentice Hall.

Loureiro, A. L. C. M. T. (2010). Porque poupamos energia? Altruísmo, ambientalismo e contexto na explicação do comportamento de poupança de energia. Tese de Doutorado. Departamento de Psicologia Social e das Organizações, ISTE- IUL, Lisboa.

Maner, J. K., \& Gailliot, M. T. (2007). Altruism and egoism: Prosocial motivations for helping depend on relationship context. European Journal of Psychology, 37, 347-358.

McCullough, M. E., Emmons, R. A., \& Tsang, J. A. (2002). The grateful disposition: A conceptual and empirical topography. Journal of Personality and Social Psychology, 82, 112-127.

McCullough, M. E., Kimeldorf, M. B., \& Cohen, A. D. (2008). An adaptation for altruism? The social causes, social effects, and social evolution of gratitude. Current
Directions in Psychological Science, 17, 281-285.

McCullough, M. E., Worthington, E. L., Jr., \& Rachal, K. C. (1997). Interpersonal forgiving in close relationships. Journal of Personality and Social Psychology, 73, 321336.

Mclean, A. M., Walker, L. J., \& Matsuba, M. K. (2004). Transcendence and the moral self: Identify integration, religion, and moral life. Journal for the Scientific Study of Religion, 43, 429-437.

Mehrabian, A., \& Epstein, N. (1972). A measure of emotional empathy. Journal of Personality, 40, 525-543.

O'Connor, L. E. (2005). Altruism and empathy-based guilt across five cultures. 34th Annual Conference of the Society for Cross-Cultural Research, Santa Fé, New Mexico.

Pasquali, L. (2003). Teoria psicométrica dos testes na psicologia e na educação. Petrópolis, RJ: Vozes.

Penner, L. A., Dovidio, J. D., Piliavin, J. A., \& Schroeder, D. A. (2005). Prosocial behavior: Multilevel perspectives. Annual Review of Psychology, 56, 365-392.

Penner, L. A., \& Finkelstein, M. A. (1998). Dispositional and structural determinants of volunteerism. Journal of Personality and Social Psychology, 74, 525-537.

Ribas Jr, R. C., Moura, M. L. S., \& Hutz, C. S. (2004). Adaptação brasileira da Escala de Desejabilidade Social de Marlowe-Crowne. Avaliação Psicológica, 3, 83-92.

Robert, B. W., \& Mroczek, D. (2008). Personality trait change in adulthood. Current Directions in Psychological Science, 17, 131-135.

Rushton, J. (2004). Genetic and environmental contributions to pro-social attitudes: A twin study of social responsibility. Proceedings of the Royal Society of London, 271, 2583-2585.

Rushton, J. P., Chrisjohn, R. D., \& Fekken, G. C. (1981). The altruistic personality and the self-report altruism scale. Personality \& Individual Differences, 2, 293-302.

Smith, T. W. (2006). Altruism and empathy in America: Trends and correlates. retirado em 18 de agosto de 2011, de http://www.norc.org/NR/rdonlyres/7EFE80C6-FD3A46F7-AF22.

Steele, W. R., Schreiber, G. B., Guiltinan, A., Nass, C., Glynn, S. A., Wright, D. J., Kessler, D., Schlumpf, K. S., Tu, Yongling, Smith, J. W., \& Garraty, G. (2008). The role of altruistic behavior, empathetic concern, and social responsibility motivation in blood donation behavior. Blood Donors and Blood Collection, 48, 43-54.

Weir, K., \& Duveen, G. (1981). Further development and validation of the prosocial bahaviour questionnaire for use by teachers. Journal of Child Psychology and Psychiatry, 22, 357-374.

Wispé, L.G. (1972). Positive forms of social behavior: An overview. Journal of Social Issues, 28, 1-19.

Worthington Jr., E. L., Sharp, C. B., \& Lerner, A. J. (2006). Interpersonal forgiveness as an example of loving one's enemies. Journal of Psychology and Theology, 34, 32-42.

Recebido em: 21/11/2013 Primeira decisão editorial em: 31/03/2015 Aceito em: 25/01/2016 